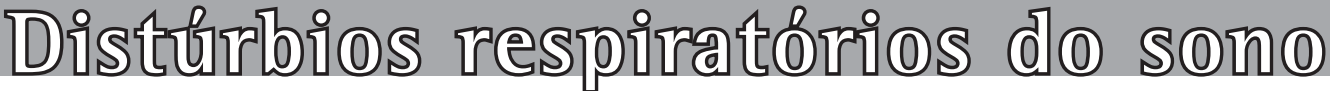

\section{Epidemiologia dos distúrbios respiratórios do sono}

\author{
Epidemiology of sleep-disordered breathing
}

Carlos Alberto de Assis Viegas

\begin{abstract}
Resumo
Os principais distúrbios respiratórios do sono, ronco e SAOS, são muito prevalentes na população geral, embora se acredite que a maioria dos casos continue não diagnosticada. Devemos estar atentos para os principais fatores de risco que favorecem o aparecimento desses distúrbios, como gênero masculino, obesidade, envelhecimento e características crânio-faciais. Da mesma forma, a presença de hipertensão arterial sistêmica, enfermidades cardiovasculares e metabólicas deve nos alertar para a possibilidade da concomitância de SAOS.
\end{abstract}

Descritores: Síndromes da apneia do sono/prevenção \& controle; Síndromes da apneia do sono/epidemiologia; Apneia do sono tipo obstrutiva/prevenção \& controle.

\begin{abstract}
The principal types of sleep-disordered breathing-snoring and obstructive sleep apnea syndrome-are highly prevalent in the general population, although it is believed that the majority of cases continue to go undiagnosed. We should be aware of the principal risk factors that favor the onset of these disorders, such as male gender, obesity, aging and craniofacial features. Similarly, systemic arterial hypertension, cardiovascular diseases and metabolic disorders should alert us to the possibility of obstructive sleep apnea syndrome.
\end{abstract}

Keywords: Sleep apnea syndromes/prevention \& control; Sleep apnea syndromes/epidemiology; Sleep apnea, obstructive/prevention \& control.

Em um inquérito realizado pela Fundação Americana do Sono, no ano de 2005, encontrou-se, na população geral, uma tendência de redução no número de horas de sono tanto durante a semana quanto nos finais de semanas. Observou-se ainda que 40\% dos entrevistados dormiam menos de $7 \mathrm{~h}$ por noite durante a semana e que 34\% apresentavam risco de ter algum distúrbio do sono. Assim sendo, os distúrbios respiratórios relacionados com o sono têm grande prevalência na população geral, sendo que, provavelmente, a maioria continue sem ter o diagnóstico realizado, mesmo sabendo-se que o diagnóstico precoce seguido de tratamento proporciona melhora clínica do paciente, bem como pode evitar consequências negativas para o organismo. ${ }^{(1)}$

0 ronco é o ruído causado pela vibração das estruturas flácidas nas vias aéreas superiores, devido a seu estreitamento, que ocorre habitualmente durante a inspiração. Ele pode acontecer em todos os estágios do sono, mas em especial durante o sono NREM. 0 ronco está presente em $30-40 \%$ da população com mais de 50 anos de idade. ${ }^{(2)}$ Em um estudo na Suécia envolvendo quase 5.000 participantes com idades entre 20 e 69 anos, encontrou-se que o ronco, se apresentando como problema, estava presente em 17,9\% da população masculina e em 7,4\% da feminina. ${ }^{(3)}$ Em outro estudo analisando uma amostra populacional com mais de 65 anos, a prevalência de ronco foi de 33\% para os homens e de 19\% para as mulheres. ${ }^{(4)}$ Estudos recentes sugerem que a obesidade central e o tabagismo são determinantes chaves para a intensidade e habitualidade dos roncos. A transição de ronco habitual para o desenvolvimento de SAOS ainda não está clara e envolvem fatores como idade, obesidade, gênero e etnicidade, bem como a presença de outras comorbidades.

No que se refere a outros distúrbios do sono, a segunda edição da Classificação Internacional de Distúrbios do Sono inclui não somente os despertares relacionados a esforços respiratórios dentro do IAH (expresso em eventos/hora de sono), mas também considera a resistência aumentada das vias aéreas superiores como parte do continuum de SAOS. ${ }^{(5)}$ Em 2002, o Sleep Heart Health Study, avaliando 5.615 homens e mulheres com idades entre 40 e 98 anos, encontrou, naqueles que relatavam ronco habitual e alto com frequentes pausas respiratórias, uma chance entre 3 e 4 vezes maior de apresentar $1 \mathrm{AH}>15 .{ }^{\left({ }^{(6)}\right.}$ Em outro estudo avaliando prospectivamente roncadores habituais, encontrou-se que $24 \%$ dos homens e $9 \%$ das mulheres tinham 
$1 \mathrm{AH}>$ 5, levando-se em consideração ou não a presença de sintomas diurnos, enquanto $9 \%$ dos homens e $4 \%$ das mulheres tinham $1 \mathrm{AH}>15{ }^{(7)}$ Lembramos que, após o início da menopausa, as mulheres apresentam uma maior prevalência de SAOS quando comparadas com mulheres antes desse início. Também foi relatada uma maior prevalência de ronco em mulheres grávidas, quando comparadas com o período antes da gravidez, e que hipertensão e pré-eclampsia se desenvolvem mais em grávidas roncadoras que em não roncadoras. ${ }^{(8)}$

A apneia do sono é a parada da respiração durante o sono, que tem como critério para sua definição a redução do fluxo inspiratório em mais de 50\% por pelo menos $10 \mathrm{~s}$. A apneia obstrutiva, causada pelo fechamento das vias aéreas superiores, apresenta parada do fluxo aéreo nasal e bucal, com manutenção do esforço ventilatório pela musculatura inspiratória, incluindo os movimentos toracoabdominais. No caso de apneias mistas, os episódios obstrutivos se iniciam como uma apneia central e continuam como apneia obstrutiva. Do ponto de vista clínico, essa situação deve receber a mesma consideração como no caso das apneias obstrutivas. ${ }^{(2)}$

A prevalência de qualquer tipo de apneia aumenta com a idade, sendo que as apneias centrais contam mais para esse aumento durante o envelhecimento. Entretanto, a gravidade da apneia do sono, indicada pelo número de eventos e pela gravidade dos valores mínimos de $\mathrm{SpO}_{2}$, diminui com a idade, ou seja, nessa situação, embora a prevalência tenda a aumentar, seu significado clínico diminui. Podemos dizer que o envelhecer está associado com uma maior prevalência de SAOS, sem estar claro, no entanto, se SAOS no idoso representa o mesmo distúrbio que no adulto de meia idade. ${ }^{(9)}$

No que se refere ao fator étnico, foi observado um aumento do risco para SAOS entre negros, latinos e asiáticos, com sugestão de que as características crânio-faciais desses grupos populacionais devem contribuir para esses maiores índices de eventos obstrutivos durante o sono. ${ }^{(10)}$ Deixamos claro que esses resultados devem sempre ser controlados para idade, índice de massa corporal e circunferência do pescoço.

Da mesma forma que o diagnóstico de SAOS deve nos sugerir a possibilidade de outros diagnósticos, a presença clínica de SAOS, obesidade e resistência à insulina é motivo para a investigação de síndrome metabólica..$^{(9)}$ Recentemente, foi sugerido que pacientes com SAOS apresentam níveis mais altos de insulina de jejum e que obesidade e distúrbios respiratórios do sono são determinantes independentes para o aumento da resistência à insulina. Além disso, a associação entre SAOS e resistência à insulina pode ser encontrada em pacientes obesos e não obesos. ${ }^{(11)}$ Sobre esse tema, lembramos que outros importantes trabalhos revelam a possível associação entre SAOS, síndrome metabólica e doença cardiovascular. ${ }^{(12,13)}$

Finalmente, salientamos que SAOS grave está associada, de forma independente, a doença arterial, inclusive periférica, ${ }^{(14)}$ e que, embora o acidente vascular encefálico não se associe isoladamente com roncos, esse está independentemente associado a hipertensão, tabagismo e índice de sonolência diurna excessiva. ${ }^{(15)}$

\section{Referências}

1. National Sleep Foundation. 2005 Sleep in America Poll. Washington, DC: National Sleep Foundation; 2006.

2. Pascual AB, Siurana EG, Sánchez PR. Tema 14: Trastornos de la respiración inducidos por el sueño. In: Universidad Pablo de Olavide y El Colegio de América Centro de Estudios Avanzados para América Latina y el Caribe, coordinators. Master en Sueño: Fisiología y Medicina. Barcelona: Viguera; 2007. p. 373-95.

3. Larsson LG, Lindberg A, Franklin KA, Lundbäck B. Gender differences in symptoms related to sleep apnea in a general population and in relation to referral to sleep clinic. Chest. 2003;124(1):204-11.

4. Enright PL, Newman AB, Wahl PW, Manolio TA, Haponik EF, Boyle PJ. Prevalence and correlates of snoring and observed apneas in 5,201 older adults. Sleep. 1996;19(7):531-8.

5. American Academy of Sleep Medicine. The International Classification of Sleep Disorders: Diagnostic and Coding Manual. Westchester: American Academy of Sleep Medicine; 2005.

6. Young T, Shahar E, Nieto FJ, Redline S, Newman AB, Gottlieb DJ, et al. Predictors of sleep-disordered breathing in community-dwelling adults: the Sleep Heart Health Study. Arch Intern Med. 2002;162(8):893900.

7. Young T, Palta M, Dempsey J, Skatrud J, Weber $\mathrm{S}$, Badr S. The occurrence of sleep-disordered breathing among middle-aged adults. N Engl J Med. 1993;328(17):1230-5.

8. Franklin KA, Holmgren PA, Jönsson F, Poromaa N, Stenlund H, Svanborg E. Snoring, pregnancy-induced hypertension, and growth retardation of the fetus. Chest. 2000;117(1):137-41.

9. Yuen KM. Epidemiology in obstructive sleep apnea. In: Kushida CA, editor. Handbook of sleep disorders. New York: Informa Healthcare; 2007. p. 27-38. 
10. Scharf SM, Seiden L, DeMore J, Carter-Pokras O. Racial differences in clinical presentation of patients with sleepdisordered breathing. Sleep Breath. 2004;8(4):173-83.

11. Ip MS, Lam B, Ng MM, Lam WK, Tsang KW, Lam KS. Obstructive sleep apnea is independently associated with insulin resistance. Am J Respir Crit Care Med. 2002;165(5):670-6.

12. Coughlin SR, Mawdsley L, Mugarza JA, Calverley PM, Wilding JP. Obstructive sleep apnoea is independently associated with an increased prevalence of metabolic syndrome. Eur Heart J. 2004;25(9):735-41.
13. Vgontzas AN, Bixler EO, Chrousos GP. Sleep apnea is a manifestation of the metabolic syndrome. Sleep Med Rev. 2005;9(3):211-24.

14. Nachtmann A, Stang A, Wang YM, Wondzinski E, Thilmann AF. Association of obstructive sleep apnea and stenotic artery disease in ischemic stroke patients. Atherosclerosis. 2003;169(2):301-7.

15. Davies DP, Rodgers H, Walshaw D, James OF, Gibson GJ. Snoring, daytime sleepiness and stroke: a case-control study of first-ever stroke. J Sleep Res. 2003;12(4):313-8.

\section{Sobre o autor}

\section{Carlos Alberto de Assis Viegas}

Professor Adjunto. Faculdade de Medicina, Universidade de Brasília, Brasília (DF) Brasil.

E-mail para contato: viegasc@uol.com.br (C. Viegas) 